\title{
Pathogenesis of diabetic nephropathy
}

\author{
Zemin Cao, Mark E Cooper*
}

\begin{abstract}
As the increasing prevalence of diabetes reaches epidemic proportions worldwide, diabetic nephropathy and associated end-stage renal failure will be an unavoidable major health burden to not only individuals with diabetes and their families, but also to the health systems both in developed and developing countries. Over the past decade, a large body of research has focused on diabetic nephropathy ranging from studies in molecular signaling, hemodynamic regulation and pharmaceutical intervention to clinical outcomes. It is likely that the pathophysiology of diabetic nephropathy involves a multifactorial interaction between metabolic and hemodynamic factors. Metabolic factors involve glucose-dependent pathways, such as advanced glycation end-products and their receptors. Hemodynamic factors include various vasoactive hormones, such as components of the renin-angiotensin system. It is likely that these metabolic and hemodynamic factors interact through shared molecular and signaling pathways, such as nuclear factor kappa-light-chain-enhancer of activated B cells and protein kinase $C$ with associated reactive oxygen species generation. It is likely that these contributing factors cause pathological damage not only to the glomerulus, in particular podocytes, but also to the tubulointerstitium. Specific inhibitors of the various pathways are now available and these emerging pharmaceutical interventions might have potential implications for the prevention and treatment of diabetic nephropathy. The mainstay of therapy remains the achievement of optimal glycemic and blood pressure control in order to slow the progression of diabetic nephropathy. Agents that interrupt the renin-angiotensin system have been shown to be particularly useful as renoprotective agents in both hypertensive and normotensive type 1 and type 2 diabetic subjects. (J Diabetes Invest, doi: 10.1111/j.2040-1124.2011.00131.x, 2011)
\end{abstract}

KEY WORDS: Angiotensin converting enzyme 2, Cell division autoantigen 1, Reactive oxygen species

\section{INTRODUCTION}

As the incidence of diabetes is increasing worldwide, diabetic nephropathy has become the main cause of chronic kidney disease in patients who require renal replacement therapy in the Western world. Furthermore, diabetic nephropathy is associated with an increased risk of cardiovascular morbidity and mortality. Intensive glycemic and blood pressure control and the use of angiotensin-converting enzyme (ACE) inhibitors and/or angiotensin II receptor blockers delay, but do not prevent, the onset and progression of diabetic nephropathy. Therefore, the disease burden of diabetic nephropathy on individuals with diabetes and the health system are enormous.

Diabetic nephropathy can be classified into different stages according to the presence of albuminuria and the degree of renal impairment. Microalbuminuria is now considered an early stage of, rather than a predictor of, diabetic nephropathy. Nevertheless, the presence of microalbuminuria is predictive for the subsequent development of severe renal impairment. Furthermore, interventions aimed at reversing microalbuminuria towards the normal range have been shown to confer beneficial effects in delaying the onset of renal impairment, and in some studies, even reversing the progression of diabetes related renal injury.

Diabetic Complications Division, Baker IDI Heart and Diabetes Institute, and Department of Immunology, Monash University, AMREP, Melbourne, Victoria, Australia

*Corresponding author. Mark E Cooper Tel.: +613-8532-1362 Fax: +613-8532-1480

E-mail address: mark.cooper@bakeridi.edu.au

Received 24 March 2011; accepted 29 March 2011
The pathogenesis and progression of diabetic nephropathy are likely to be as a result of interactions between metabolic and hemodynamic pathways, which are often disturbed in the setting of diabetes. Since major reviews on this topic by our group over a decade ago ${ }^{1,2}$, there have been major advances in this area. It is likely that the metabolic and hemodynamic abnormalities seen in diabetes interact with each other and pathways linked to reactive oxygen species (ROS) generation. Gene regulation and activation of transcription factors are influenced by interactions among metabolic stimuli, hemodynamic factors and various ROS in diabetes. The consequences of molecular activation and inhibition of the various pathways lead to functional and structural changes that clinically become manifest as diabetic nephropathy, characterized by increasing albuminuria and declining renal function (Figure 1).

In the present review, we will focus on a number of pathways/factors that have been shown to contribute to renal injury in various experimental models of nephropathy, including studies by our group.

\section{ANGIOTENSIN CONVERTING ENZYME 2 AS A MEDIATOR IN RENAL INJURY}

Blockade of the renin-angiotensin system (RAS) is considered to be among the most important interventions for the prevention and management of diabetic kidney disease. The RAS blockers, either inhibiting ACE, blocking activation of the angiotensin type $1\left(\mathrm{AT}_{1}\right)$ receptor or directly inhibiting the upstream 

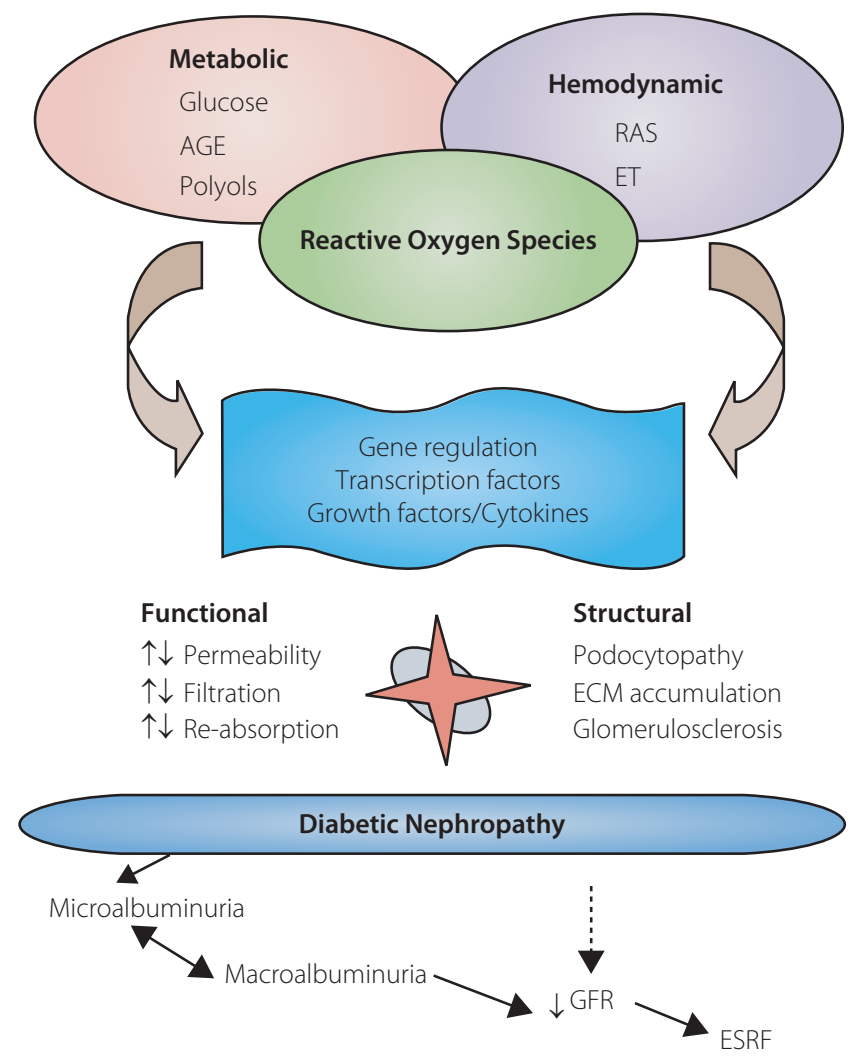

Figure $\mathbf{1}$ | Schema of pathogenesis of diabetic nephropathy. The pathogenesis of diabetic nephropathy is likely to be as a result of metabolic and hemodynamic abnormalities, as seen in diabetes, interacting with each other and with various reactive oxygen species-dependent pathways. Both gene regulation and activation of transcription factors are influenced by the interactions between metabolic stimuli, hemodynamic factors and reactive oxygen species generation in diabetes. The consequences of this molecular activation or inhibition are functional and structural changes leading to the classical hallmarks of diabetic nephropathy. AGE, advanced glycation end-products; ECM, extracellular protein production; ESRF, end-stage renal failure; ET, endothelin; GFR, glomerular filtration rate.

enzyme, renin, are effective in attenuating renal damage in diabetes. Historically, actions of the RAS blockers have been linked to reduced signaling through angiotensin II-dependent pathways.

Angiotensin converting enzyme 2 (ACE2) is an exopeptidase that catalyses the conversion of angiotensin I to the nonapeptide angiotensin 1-9, or the conversion of angiotensin II to angiotensin 1-7. In the kidney, angiotensin 1-7 is largely derived from the degradation of angiotensin II by the zinc-dependent carboxypeptidase $\mathrm{ACE} 2^{3,4}$. ACE2 expression has been shown to be present in the kidney, heart and blood vessels ${ }^{5-7}$.

ACE2 is expressed in renal tubular cells, as assessed by immumohistochemistry ${ }^{8}$. This ACE2 protein expression is associated with ACE2 gene expression, renal cortical ACE2 enzymatic activity and plasma ACE2 activity. Administration of
MLN-4760, an ACE2 inhibitor, in mice is associated with inhibition of renal ACE2 activity.

The pattern of ACE2 expression in the kidney is significantly modified after the induction of diabetes in experimental animals ${ }^{8}$. Induction of diabetes in mice is associated with a reduction in renal ACE2 expression and a concomitant decrease in angiotensin 1-7 levels ${ }^{8}$. Paradoxically and yet not fully explained, plasma ACE2 activity is significantly elevated after administration of the ACE2 inhibitor, MLN-4760. Administration of an ACE inhibitor, perindopril, although this is not a direct ACE2 inhibitor, or the ACE2 inhibitor, MLN-4760, are both associated with reductions in renal ACE2 expression and plasma ACE2 activity, as well as a decrease in renal cortical angiotensin 1-7 concentrations in diabetic mice. However, there are different effects between an ACE and an ACE2 inhibitor on albuminuria, blood pressure and creatinine clearance in diabetic mice. Diabetic mice treated with an ACE inhibitor have reduced urinary albumin excretion, whereas diabetic rats treated with an ACE2 inhibitor have increased albuminuria. Furthermore, diabetic mice treated with an ACE inhibitor have reduced blood pressure, whereas ACE2 inhibitor treated diabetic mice show increased blood pressure. In addition, ACE inhibitor treatment has no effects on the elevated glomerular filtration rate (GFR), seen in diabetic mice, whereas ACE2 inhibitor treatment reduces this hyperfiltration. Finally, antiproteinuric and hypotensive effects of ACE inhibitor are lost when an ACE inhibitor is combined with an ACE2 inhibitor in diabetic mice ${ }^{8}$. Consistent with the results seen with pharmacological ACE2 inhibition, the effects of ACE2 inhibition using a genetic approach on albuminuria, blood pressure and creatinine clearance have been confirmed in ACE2 knockout mice ${ }^{8}$.

These recent findings linking ACE2 to diabetic nephropathy suggest that the ACE2-angiotensin 1-7 axis might have specific effects on the pathology of diabetic nephropathy. Furthermore, it appears that at least some the systemic effects of RAS blockade might be mediated by angiotensin 1-7, a potent vasodilator, with actions that antagonize or counteract those of angiotensin II. Furthermore, it is likely that the role of ACE2 in diabetic nephropathy is complex, with its effects varying according to the different renal sites where ACE2 might have an important function ${ }^{9,10}$.

\section{CELL DIVISION AUTOANTIGEN 1 IN MEDIATING TRANSFORMING GROWTH FACTOR- $\beta$ DOWNSTREAM SIGNALING AND EXTRACELLULAR PROTEIN PRODUCTION}

Excess accumulation of extracellular protein production (ECM) in the kidney is increasingly considered to play an important role in the development and progression of diabetic nephropathy. Transforming growth factor- $\beta$ (TGF- $\beta$ ) has been viewed as a key player in mediating the profibrotic effect of various pathological stimuli, such as hyperglycemia and angiotensin II, most of these functions enhanced in the diabetic milieu, thus leading to excess ECM accumulation in the diabetic kidney. 
TGF- $\beta$ also upregulates another growth factor, connective tissue growth factor (CTGF), through various signaling pathways involving specific molecules known as Smads.

Cell division autoantigen 1 (CDA1) is a molecule identified by Chai et al. ${ }^{11}$ and named to reflect the specific finding that CDA1 arrested cell growth when overexpressed in HeLa cells. Its antiproliferative action is supported by further findings by our group, as well as by others ${ }^{12-14}$. CDA1, encoded by TSPYL2 on the $\mathrm{X}$ chromosome, shares antiproliferative and profibrotic properties with TGF- $\beta$. It inhibits cell growth through $\mathrm{p} 53$, pERK1/2 and p21-mediated pathways, and is implicated in tumorigenesis and the DNA damage response ${ }^{15}$. Its profibrotic properties are mediated through cross-talk with TGF- $\beta$, which results in upregulation of extracellular matrix proteins. We have obtained a large body of data showing that CDA1 modulates the TGF- $\beta$ signaling pathway through TGF- $\beta$ receptor I and Smad $3{ }^{16}$. This TGF- $\beta$ signaling through Smad 3 , which can also be activated by other stimuli, such as angiotensin II, regulates ECM production. Schema of TGF- $\beta$ induction of tissue fibrosis has been reviewed elsewhere ${ }^{15}$.

More recently, we have shown that CDA1 levels were elevated in the kidneys from two animal models with increased renal $\mathrm{ECM}^{17}$. The localization of CDA1 to the tubular cells and podocytes in the human kidney was similar to that seen in rodents. Overexpression and siRNA knockdown of CDA1 resulted in a dose-dependent increase or decrease in gene expression of TGF- $\beta$, TGF- $\beta$ receptors, CTGF, collagens type I, III, and IV, and fibronectin in a human kidney cell line known as HK-2 ${ }^{12}$. CDA1 siRNA knockdown markedly attenuated, whereas overexpression of CDA1 increased, TGF- $\beta$ signaling. CDA1 and TGF- $\beta$ together had a strong synergy in stimulating TGF- $\beta$ signaling and increasing expression of various target genes. CDA1 knockdown effectively blocked the effect of TGF- $\beta$ in stimulating expression of collagen genes. These activities of CDA1 are attributed to its ability to modulate the TGF- $\beta$ type I, but not the type II, receptor, leading to increased phosphorylation of Smad3 and activation of the ERK/MAPK pathway. Furthermore, the Smad3 inhibitor, SIS3, was shown to markedly attenuate the activity of CDA1 to stimulate TGF- $\beta$ signaling, as well as reducing gene expression of collagens I, III and IV.

These in vitro and in vivo findings provide evidence that CDA1 plays a critical role in TGF- $\beta$ signaling in the kidney and is likely to be an appropriate target to reduce renal ECM accumulation in disorders such as diabetic nephropathy.

\section{PODOCYTE ABNORMALITY AND ALBUMINURIA}

The importance of glomerular abnormalities in pathogenesis of diabetic nephropathy has been a focus of ongoing research. An increase in glomerular filtration, in association with albuminuria, has been a hallmark of early diabetic nephropathy.

Podocytes (or visceral epithelial cells) are highly specialized cells of neuroepithelial origin that wrap around the capillaries of the glomerulus. The long processes of the podocytes wrap around the capillaries and between these processes are small slits in which contain a slit diaphragm. An increasing number of proteins have been identified to be present in these foot projections that wrap around the capillaries.

Nephrin is a zipper-like protein that plays a functional role in the structure of the slit diaphragm. The spaces between the teeth of the zipper allow, in a selective manner, small molecules, such as glucose and water, to traverse, but are too small to allow large proteins to cross. Indeed, defects in nephrin have been reported to be responsible for the massive albuminuria that occurs in the Finnish type of congenital nephritic syndrome and kidney failure.

There is now an increasing body of evidence to suggest that nephrin could play a key role in the function of the glomerular filtration barrier and the development of proteinuria. In diabetes, early flattening and retraction of the foot processes are associated with thickening of the glomerular basement membrane. Indeed, in diabetic rats, there is a reduction in the number of filtration slits with an associated reduction in nephrin expression ${ }^{18}$.

Nephrin is increasingly expressed in the developmental kidney from day 5 after birth to adulthood in rats ${ }^{19}$. However, nephrin expression is lower in the kidneys from spontaneously hypertensive rats (SHR) in an association with hypertension and proteinuria, than seen in the kidneys from normotensive Wistar-Kyoto rats $(\mathrm{WKY})^{19}$. This relationship between nephrin expression and albuminuria in normotensive and hypertensive diabetic rats was studied by our group ${ }^{20}$. Nephrin gene expression transiently rose at 8 weeks in the diabetic SHR and then significantly decreased after 16 and 24 weeks. This reduction in glomerular nephrin gene expression was associated with increasing albuminuria at 16 and 24 weeks in these diabetic SHR. There were no significant changes in nephrin gene expression observed in normotensive diabetic WKY rats and this lack of an effect was seen in the context of much less albuminuria in these WKY rats ${ }^{20}$.

Renal nephrin expression was also reduced in a non-diabetic renal injury model, the subtotal nephrectomy model. In this model, the subtotal nephrectomy (removal of one kidney and ablation of two-thirds of the remaining kidney) in rats was associated with systemic hypertension, renal fibrosis and massive proteinuria ${ }^{21}$. These findings further emphasize the pivotal role of slit diaphragm proteins, such as nephrin, in the pathogenesis of albuminuria and proteinuria, either in a diabetic or nondiabetic milieu.

Renal nephrin expression is not only closely associated with the development of albuminuria, as observed in an experimental model of diabetes and hypertension, but also is affected by various treatments. Indeed, it is likely that the renal protection conferred by RAS blockers is at least in part related to the effects of these drugs on podocyte slit diagram proteins such as nephrin ${ }^{18}$.

In long-term diabetes (32 weeks) there was a reduction in both gene and protein expression of nephrin within the kidneys in these rats ${ }^{18}$. These changes in nephrin levels were prevented by the angiotensin II antagonist, irbesartan, suggesting a potential novel mechanism to at least in part explain the antiproteinuric effect of agents that interrupt the RAS ${ }^{18}$. Similar effects were also 
observed with another angiotensin II antagonist, valsartan ${ }^{22}$. However, treatment with calcium channel blockers (amlodipine or verapamil), another class of antihypertensive agents, have no effects on nephrin expression and proteinuria, even though these non-RAS oriented treatments had similar blood pressure lowering effects to the angiotensin II antagonists ${ }^{22}$.

\section{OXIDATIVE STRESS AND DIABETIC RENAL INJURY}

Hyperglycemia induces vascular injury through complex overlapping pathways, including formation of advanced glycation end-products (AGE), activation of protein kinase C (PKC) and generation of ROS. There is growing evidence suggesting that ROS might play an important role in the initiation and progression of diabetic nephropathy ${ }^{23}$. The effects of anti-oxidant therapy have been shown in animal studies, although convincing evidence for clinical efficacy is still lacking.

Excessive production of ROS through nicotinamide adenine dinucleotide phosphate (NADPH) oxidase (Nox) has been implicated in the pathogenesis of diabetic nephropathy. Diabetes-induced translocation of PKC, specifically PKC- $\alpha$ to renal membranes, was associated with increased NADPH-dependent superoxide production and elevated renal, serum and urinary vascular endothelial growth factor (VEGF) concentrations ${ }^{24}$. Blockade of Nox with apocynin attenuated diabetes-associated increases in albuminuria and glomerulosclerosis ${ }^{24}$. In both diabetic rodents and in AGE-treated mesangial cells, blockade of Nox or PKC- $\alpha$ attenuated cytosolic superoxide generation and PKC activation, as well as increased VEGF. Furthermore, renal extracellular matrix accumulation of fibronectin and collagen IV was decreased by the Nox inhibitor apocynin. Thus, it is possible that inhibition of Nox might provide a novel therapeutic target for diabetic nephropathy, although more selective Nox inhibitors might be required ${ }^{23,24}$.

\section{ACKNOWLEDGMENTS}

The authors' research was funded by the National Health and Medical Research Council of Australia, the National Heart Foundation of Australia and the Juvenile Diabetes Research Foundation. MEC is an Australian Fellow from the National Health and Medical Research Council of Australia. ZC and MEC have an international patent at National Phase with the Baker IDI Heart and Diabetes Institute and Dia-B Tech, Melbourne, Australia: 'Methods and compositions for treating disorders of the extracellular matrix'. Otherwise, there is no duality of interest associated with this manuscript.

\section{REFERENCES}

1. Cooper ME. Pathogenesis, prevention, and treatment of diabetic nephropathy. Lancet 1998; 352: 213-219.

2. Cooper ME. Interaction of metabolic and haemodynamic factors in mediating experimental diabetic nephropathy. Diabetologia 2001; 44: 1957-1972.

3. Tikellis C, Bernardi S, Burns WC. Angiotensin-converting enzyme 2 is a key modulator of the renin-angiotensin system in cardiovascular and renal disease. Curr Opin Nephrol Hypertens 2010; 20: 62-68.

4. Burrell $L M$, Johnston $C 1$, Tikellis $C$, et al. ACE2, a new regulator of the renin-angiotensin system. Trends Endocrinol Metab 2004; 15: 166-169.

5. Koitka A, Cooper ME, Thomas MC, et al. Angiotensin converting enzyme 2 in the kidney. Clin Exp Pharmacol Physiol 2008; 35: 420-425.

6. Thomas MC, Pickering RJ, Tsorotes D, et al. Genetic Ace2 deficiency accentuates vascular inflammation and atherosclerosis in the ApoE knockout mouse. Circ Res 2010; 107: 888-897.

7. Burrell LM, Risvanis J, Kubota E, et al. Myocardial infarction increases ACE2 expression in rat and humans. Eur Heart J 2005; 26: 369-375; discussion 322-324.

8. Tikellis C, Bialkowski K, Pete J, et al. ACE2 deficiency modifies renoprotection afforded by ACE inhibition in experimental diabetes. Diabetes 2008; 57: 1018-1025.

9. Tikellis $\mathrm{C}$, Johnston $\mathrm{Cl}$, Forbes JM, et al. Characterization of renal angiotensin-converting enzyme 2 in diabetic nephropathy. Hypertension 2003; 41: 392-397.

10. Frojdo S, Sjolind L, Parkkonen $M$, et al. Polymorphisms in the gene encoding angiotensin I converting enzyme 2 and diabetic nephropathy. Diabetologia 2005; 48: 2278-2281.

11. Chai Z, Sarcevic B, Mawson A, et al. SET-related cell division autoantigen-1 (CDA1) arrests cell growth. J Biol Chem 2001; 276: 33665-33674.

12. Tu Y, Wu W, Wu T, et al. Antiproliferative autoantigen CDA1 transcriptionally up-regulates p21(Waf1/Cip1) by activating p53 and MEK/ERK1/2 MAPK pathways. J Biol Chem 2007; 282: $11722-11731$.

13. Ito T, Tsukumo S, Suzuki N, et al. A constitutively active arylhydrocarbon receptor induces growth inhibition of jurkat $T$ cells through changes in the expression of genes related to apoptosis and cell cycle arrest. J Biol Chem 2004; 279: 25204-25210.

14. Radke JR, Donald RG, Eibs A, et al. Changes in the expression of human cell division autoantigen-1 influence Toxoplasma gondii growth and development. PLoS Pathog 2006; 2: e105.

15. Toh BH, Tu Y, Cao Z, et al. Role of cell division autoantigen 1 (CDA1) in cell proliferation and fibrosis. Genes 2010; 1: 335348.

16. Pham Y, Tu Y, Wu T, et al. Cell division autoantigen 1 plays a profibrotic role by modulating downstream signalling of TGF-beta in a murine diabetic model of atherosclerosis. Diabetologia 2010; 53: 170-179.

17. Tu Y, Wu T, Dai A, et al. Cell division autoantigen 1 enhances signaling and the profibrotic effects of transforming growth factor-beta in diabetic nephropathy. Kidney Int 2011; 79: 199-209.

18. Bonnet F, Cooper ME, Kawachi $\mathrm{H}$, et al. Irbesartan normalises the deficiency in glomerular nephrin expression in a model of diabetes and hypertension. Diabetologia 2001; 44: 874877. 
19. Bonnet F, Tikellis C, Kawachi H, et al. Nephrin expression in the post-natal developing kidney in normotensive and hypertensive rats. Clin Exp Hypertens 2002; 24: 371381.

20. Forbes JM, Bonnet F, Russo LM, et al. Modulation of nephrin in the diabetic kidney: association with systemic hypertension and increasing albuminuria. J Hypertens 2002; 20: 985-992.

21. Cao Z, Bonnet F, Candido R, et al. Angiotensin type 2 receptor antagonism confers renal protection in a rat model of progressive renal injury. J Am Soc Nephrol 2002; 13: 1773-1787.
22. Davis BJ, Cao Z, de Gasparo M, et al. Disparate effects of angiotensin II antagonists and calcium channel blockers on albuminuria in experimental diabetes and hypertension: potential role of nephrin. J Hypertens 2003; 21: 209-216.

23. Forbes JM, Coughlan MT, Cooper ME. Oxidative stress as a major culprit in kidney disease in diabetes. Diabetes 2008; 57: 1446-1454.

24. Thallas-Bonke V, Thorpe SR, Coughlan MT, et al. Inhibition of NADPH oxidase prevents advanced glycation end productmediated damage in diabetic nephropathy through a protein kinase C-alpha-dependent pathway. Diabetes 2008; 57: 460-469. 